Vol. 53, z $1-2000$

s. $75-86$

\title{
Flora of field plantings and parks and its origin
}

\section{CZESLAW HOLDYŃSKI, MIECZYSLAWA ALDONA FENYK}

\begin{abstract}
Departament of Botany and Nature Protection, Warmia and Mazury University in Olsztyn, 10-727 Olsztyn, Plac kódzki 1
\end{abstract}

(Received 17.01.2000)

\section{Summary}

The object of the studies presented in this paper was the flora of field plantings of different origin. The vascular flora of the plantings examined comprises 254 taxons, including 53 trees and shrubs, and 201 herbaceous vascular plants. The flora is rich and diverse. In $26.8 \%$ of taxons it is presents in all types of plantings. More than half of the registered species occurs once or twice only in all 23 planting groups studied.

A detailed analysis of the flora examined shows that field plantings in agricultural landscapes may, in a small number of cases, constitute a source of weeds diaspores propagating in fields.

Key words: field plantings, flora, diversity, synanthropization.

\section{INTRODUCTION}

Agricultural landscapes are components of various vegetation complexes. The following vegetation types predominate here: segetal vegetation with spatially scattered complexes of meadow and pasture vegetation, aquatic and rush vegetation of small ponds in fields, forest vegetation and shrubs characteristic of field plantings, ruderals that can be found on roadsides, in built-up areas, semi-natural sandy and xerothermic grass, natural communities which form ecotone zones with the above-mentioned ones. Each spatial unit of a landscape is characterized by certain distinguishing features, such as: the origin of those complexes, their vegetation, area and shape, distance between them, degree of isolation, internal biotopic, floral and phytosociological diversity, nature and intensity of man's economic activities. 
From the agricultural perspective, it seems important to what degree the structure of vegetation complexes in a given landscape influences the species and quantitative composition of weeds in fields under cultivation, and vice versa. This paper constitutes an attempt to answer this question. It deals with vegetation complexes present in different types of field plantings in selected areas, compared with the segetal flora of North-Eastern Poland.

\section{RESEARCH AREA AND MATERIAL}

The paper is based on floral research conducted in the years 1996 - 1997 in object 'Wola' located in the Mragowskie Lake District, in the zone of high relief characteristic of end moraine. A plan of anti-erosion landscape management was implemented in this area in the years 1966 - 1967 and 1975. This plan included tree planting according to the concept elaborated by N i e w i a d o m s k i and G r a b a r c z y k (1977) - Figure 1. Plantings constitute $11.8 \%$ ( 31.9 ha) of the total area ( 270.8 ha). 23 groups of plantings studied (the so called 'islands', clumps of trees, tree belts) were divided into 3 types, i.e. 'spontaneous' (natural) plantings - which are a result of natural succession (7 objects), trees planted in the years 1966 - 1967 and poplar plantations from 1975 (10 objects altogether), plantings in the form of parks (a.park near a farm, an abandoned Evangelic park) and plantings located at the sites of deserted farms (6 objects altogether). The flora examined was subjected to an analysis based on various criteria, such as: geographical and historical origin - following the concept formulated by T h e 11 u n g (1915 and 1918-1919) and modified by K or n a ś (1968), origin of apophytes, characteristics of flora within the 3 types of plantings mentioned above, species structure of flora in the plantings studied, compared with the segetal flora of North-Eastern Poland (K o r $\mathrm{n}$ i a k 1992), the presence of basic life-forms according to R a u n k i a e r (1934) and modified by S z a f e r (1964).

The nomenclature used for vascular plants is based on the 'Vascular Plants of Poland - a Checklist' (M i r e k et al. 1995).

\section{RESULTS AND DISCUSSION}

The vascular flora of the plantings examined comprises 254 taxons, including 53 trees and shrubs (Table 1), and 201 herbaceous vascular plants (Table 2). Only part (17 taxons) of the rich dendroflora comes from plantings ( $\mathrm{H}$ o $ł \mathrm{~d}$ y n s k i, F e n y k 1998). The remaining trees and shrubs enriched the plantings in a 'spontaneous', usually anemochoric, way. They could also be a component of spontaneously formed groups of shrubs or wet leafy trees (alders) and riverside plantings, situated mainly in depressions. Sometimes these are also trees and shrubs growing near farms, e.g. Prunus domestica, Pyrus communis, Cerasus austera, Philadelphus coronarius, Rosa pimpinellifolia and other. 
The flora of herbaceous vascular plants found in the types of plantings studied and presented in Table 2 is characterized by numerous species and great diversity. In $26.8 \%$ of taxa it occurs in all types of plantings. More than half of the registered species occurs once or twice only in all planting groups studied.

The floral distinction is clearly characterized by the geographical and historical origin of the flora discussed, presented in Figures 2 and 3. Apophytes constitute the largest plant group (86.4) in all the plantings. Forest and shrub apophytes predominate here, meadow ones are less frequent (Figure 3). Similar proportions were noted by R a t y ń s k a and S z w e d (1998) in the Wielkopolska region. Despite the close vicinity of fields and small areas of tree clumps - which makes them all ecotone zones - archaeophytes are rare and sparse here (only 16 taxons were found: Convolvulus arvensis, Vicia angustifolia, Vicia tetrasperma, Vicia hirsuta, Fallopia convolvulus, Lamium purpureum, Sonchus oleraceus and other) - Figure 2.

The spectrum of plant life-forms in plantings is represented by all forms of plant adaptation to adverse environmental conditions (Figure 4). However, it is completely different from the spectrum characteristic of segetal communities (e.g. K o r n i a k 1992). Apart from dendroflora belonging to phanerophytes and chamaephytes, hemicryptophytes, geophytes and hydrophytes predominate among herbaceous plants. Annual seed plants (therophytes), which constitute over 60\% - 70\% of the other segetal communities in plantings are represented by 28 taxa $(11.1 \%)$ only. This confirms the rule that the disappearance of therophytes is connected with the presence of community complexes which ecological structure is transformed to a lower and lower degree.

Assuming that the segetal flora of a given area includes taxons which are more or less frequently, or even sporadically, noted as weeds of production fields, the flora of examined plantings was compared with the segetal flora of North-Eastern Poland (K o r n i a k 1992). It turned out that $30.7 \%$, i.e. 111 species, which were registered as segetal plants in North-Estern Poland (Figure 5). Only 22 out of that segetal plant species noted in those plantings may be considered very common and common in fields. Those are archaeophytes which occur very sporadically in plantings (Convolvulus arvensis, Viola arvensis, Vicia angustifolia, Vicia hirsuta, Lamium purpureum, Fallopia convolvulus and Sonchus oleraceus). Due to their insignificant number, they practically do not constitute a source of field infestation with weeds. This group (22 taxons) also includes apophytes - more frequent in plantings - such as Galium aparine, Stellaria media, Cirsium arvense, Agropyron repens, Artemisia vulgaris, Equisetum arvense, and - sporadically - Plantago major, Galeopsis tetrahit, Chenopodium album, Poa annua, Taraxacum officinale, Vicia villosa, Tussilago farfara, Rumex crispus, Melandrium album. The other herbaceous species noted in the plantings discussed (90 taxons) are known as the so called non-synanthropic spontaneophytes. Forest species characteristic of dry-ground habitats, belonging to class Querco-Fagetea, predominate in this group (H o ld y ń s k i, F e n y k 1998).

Taking into consideration the occurrence frequency of common segetal species in different types of plantings, they occur most often in trees planted by man (Figure 6). Their number was here by ca. 3 times higher than in 'spontaneous' plantings and anthropogenic plantings like parks, cemeteries, deserted habitats. This suggests that in the case of trees planted by man segetal species are the remains of the former system 
of agricultural use of those habitats, while in that of 'spontaneous' (natural) plantings they are a symptom of synanthropization of those plant associations. W a s i ło w s k a (1998), who studied the problems connected with synanthropization of forest islands in the catchment basin of the Jorka river, reached similar conclusions.

\section{CONCLUSIONS}

The flora of field plantings, as a component of agricultural landscapes, may - in a small number of cases - constitute a source of weed diaspores propagating in fields. This is confirmed by very rare occurrence of synanthropic (segetal) therophytes and archaeophytes. Among the species considered apophytes in fields and present in the plantings examined, only some can disseminate in fields. These are: Galium aparine, Stellaria media, Agropyron repens, Artemisia vulgaris. The other apophytes occur only sporadically in fields or the plantings examined, so there is no possibility of their dissemination in agricultural ecosystems.

\section{REFERENCES}

H o ld y ń s k i Cz., F e n y k M. A., 1998. Rola fitocenotyczna zadrzewień przeciwerozyjnych w obiekcie Wola na Pojezierzu Mragowskim. Bibliotheca Fragmenta Agronomica 5. Wyd. ART Olsztyn: 339-350.

K o r n a ś J., 1968. Geograficzno-historyczna klasyfikacja roślin synantropijnych. Mat. Zakł. Fitosoc. Stos. UW: 25:33-41.

K o r n i a k T.,1992. Flora segetalna północno-wschodniej Polski, jej przestrzenne zróżnicowanie i współczesne przemiany. Acta Academiae Agriculturae ac Technicae Olstenensis, No 53, Supplementum A. Wyd. ART Olsztyn.

M i r e k Z., et al.,1995. Vascular Plants of Poland - a Checklist. Polish Botanical Studies, Guidebook Series, 15: 1: 303.

N i e w i a d o m s k i W., G r a b a r c z y k S., 1977. Struktura użytkowania ziemi jako czynnik ochrony gleb przed erozją wodna. Zesz. Problemowe Postępów Nauk Roln. ART-Olsztyn, z.193, s. 135-155.

R a t y ń s k a H., S z w e d W., 1998. Wpływ struktury krajobrazu na zróżnicowanie szaty roślinnej i zgrupowań zwierząt. Ekologia wysp leśnych. Wyd. Uczelniane WSP w Bydgoszczy: 193-198.

R a u n k i a e r C., 1934. The life form of plants. Oxford University Press, Oxford.

$\mathrm{S} z$ a f e $\mathrm{r}$ W., 1964. Ogólna geografia roślin. PWN, Warszawa.

$\mathrm{T}$ h e $1 \mathrm{l}$ u n g A., 1915. Pflanzenwanderungen unter dem Einfluss des Menschen. Beibl. Englers Bot. Jahrb. $53(9 / 5) \mathrm{Nr} 116$.

T h e 11 u n g A., 1918-1919. Zur Terminologie der Adventiv- und Ruderalflora. Allg. Bot. Z. Syst. Karlsruhe 24, 36-42.

W a s i ł o w s k a A., 1998. Problemy synantropizacji wysp leśnych w krajobrazie rolniczym. Ekologia wysp leśnych. Wyd. Uczelniane WSP w Bydgoszczy: 279-292. 


\section{Flora zadrzewień śródpolnych i parków oraz jej pochodzenie}

\section{Streszczenie}

Przedmiotem badań niniejszego opracowania była flora zadrzewień śródpolnych różnego pochodzenia. Flora naczyniowa badanych zadrzewień liczy 254 taksony, w tym 53 stanowią drzewa i krzewy oraz 201 zielne rośliny naczyniowe. Bogata flora jest silnie zróżnicowana. Prawie 27,0\% taksonów występuje we wszystkich typach zadrzewień. Ponad połowa zarejestrowanych taksonów występuje z częstością nie większa jak 1-2 krotnie we wszystkich 23 badanych zadrzewieniach.

Wnikliwa analiza badanej flory wykazała, że zadrzewienia śródpolne w krajobrazie rolniczym w nieznacznym stopniu mogą być źródłem diaspor chwastów przenikajacych na pola.

\section{Table 1}

Flora of trees and shrubs and the frequency of their occurrence in different types of field plantings

\begin{tabular}{|c|c|c|c|c|c|}
\hline \multirow[b]{2}{*}{ No } & \multirow[b]{2}{*}{ Species } & \multicolumn{4}{|c|}{ Origin of plantings and the frequency of trees and shrubs occurrence } \\
\hline & & $\begin{array}{c}\text { natural } \\
\text { plantings }\end{array}$ & $\begin{array}{c}\text { trees planted } \\
\text { by man }\end{array}$ & $\begin{array}{c}\text { parks and other } \\
\text { types of } \\
\text { plantings }\end{array}$ & $\begin{array}{l}\text { occurrence } \\
\text { stability }\end{array}$ \\
\hline \multicolumn{2}{|c|}{ Number of research objects } & 7 & 10 & 6 & $100 \%$ \\
\hline \multicolumn{6}{|c|}{ Trees } \\
\hline 1. & Acer platanoides & 7 & 10 & 6 & 100,0 \\
\hline 2. & Quercus robur & 7 & 8 & 3 & 78,2 \\
\hline 3. & Sorbus aucuparia & 7 & 8 & 2 & 73,9 \\
\hline 4. & Betula pendula & 6 & 8 & 3 & 73,9 \\
\hline 5. & Picea abies & 3 & 8 & 2 & 56,5 \\
\hline 6. & Tilia cordata & 1 & 6 & 6 & 56,5 \\
\hline 7. & Salix caprea & 6 & 5 & 2 & 56,5 \\
\hline 8. & Alnus glutinosa & 6 & 5 & 1 & 52,1 \\
\hline 9. & Acer pseudoplatanus & 6 & 3 & 1 & 43,4 \\
\hline 10. & Fraxinus excelsior & 3 & 4 & 3 & 43,4 \\
\hline 11. & Populus tremula & 4 & 2 & 3 & 39,1 \\
\hline 12. & Carpinus betulus & 3 & 5 & 1 & 39,1 \\
\hline 13. & Malus domestica & 1 & 2 & 5 & 34,7 \\
\hline 14. & Pyrus communis & 1 & 3 & 3 & 30,4 \\
\hline 15. & Prunus insititia & 1 & 4 & 2 & 30,4 \\
\hline 16. & Fagus sylvatica & 3 & 2 & 1 & 26,0 \\
\hline 17. & Ulmus minor & 1 & 4 & - & 21,7 \\
\hline 18. & Padus avium & 1 & 1 & - & 8,5 \\
\hline 19. & Pinus sylvestris & - & 6 & 1 & 30,4 \\
\hline 20. & Prunus domestica & - & 1 & 3 & 17,4 \\
\hline 21. & Betula pubescens & 3 & - & - & 13,0 \\
\hline 22. & Salix fragilis & 1 & - & - & 4,3 \\
\hline 23. & Ulmus glabra & 1 & - & - & 4,3 \\
\hline 24. & Larix decidua & - & 7 & - & 30,4 \\
\hline 25. & Quercus rubra & - & 5 & - & 21,7 \\
\hline 26. & Populus $x$ hybryda & - & 2 & - & 8,5 \\
\hline
\end{tabular}




\begin{tabular}{|c|l|c|c|c|c|}
\hline \multicolumn{5}{|c|}{ Shrubs } \\
\hline 27. & Crataegus monogyna & $\mathbf{4}$ & $\mathbf{7}$ & $\mathbf{3}$ & $\mathbf{6 1 , 0}$ \\
\hline 28. & Sambucus nigra & $\mathbf{4}$ & $\mathbf{7}$ & $\mathbf{3}$ & $\mathbf{6 0 , 8}$ \\
\hline 29. & Corylus avellana & $\mathbf{3}$ & $\mathbf{4}$ & $\mathbf{1}$ & $\mathbf{3 4 , 7}$ \\
\hline 30. & Rosa canina & $\mathbf{1}$ & $\mathbf{3}$ & $\mathbf{1}$ & $\mathbf{2 1 , 7}$ \\
\hline 31. & Ribes nigrum & 7 & 2 & - & 39,1 \\
\hline 32. & Rosa sherardii & 1 & 2 & - & 13,0 \\
\hline 33. & Salix cinerea & 1 & 2 & - & 13,0 \\
\hline 34. & Symphoricarpos albus & - & 5 & 2 & 30,4 \\
\hline 35. & Cerasus austera & - & 1 & 5 & 26,0 \\
\hline 36. & Frangula alnus & 4 & - & - & 17,4 \\
\hline 37. & Sambucus racemosa & 4 & - & - & 17,4 \\
\hline 38. & Lonicera xylosteum & 1 & - & - & 4,3 \\
\hline 39. & Salix aurita & 3 & - & - & 13,0 \\
\hline 40. & Rosa villosa & 1 & - & - & 4,3 \\
\hline 41. & Euonymus europaea & 1 & - & - & 4,3 \\
\hline 42. & Chaenomeles lagenaria & - & 6 & - & 26,0 \\
\hline 43. & Rosa rugosa & - & 3 & - & 13,0 \\
\hline 44. & Spiraea salicifolia & - & 3 & - & 13,0 \\
\hline 45. & Caragana arborescens & - & 2 & - & 8,5 \\
\hline 46. & Prunus spinosa & - & 2 & - & 8,5 \\
\hline 47. & Rosa glauca & - & 2 & - & 8,5 \\
\hline 48. & Cornus sanguinea & - & 1 & - & 4,3 \\
\hline 49. & Daphne mezereum & - & 1 & - & 4,3 \\
\hline 50. & Hippophaë rhamnoides & - & 1 & - & 4,3 \\
\hline 51. & Viburnum opulus & - & 1 & - & 4,3 \\
\hline 52. & Philadelphus coronarius & - & - & 1 & 4,3 \\
\hline 53. & Rosa pimpinellifolia & - & - & 1 & 4,3 \\
\hline & & & & & \\
\hline
\end{tabular}

Table 2

Flora of herbaceous vascular plants and the frequency of their occurrence in different types of field plantings

\begin{tabular}{|c|c|c|c|c|c|}
\hline \multirow[b]{2}{*}{ No } & \multirow[b]{2}{*}{ Species } & \multicolumn{4}{|c|}{$\begin{array}{l}\text { Origin of plantings and the frequency of herbaceus vascular plants } \\
\qquad \text { occurrence }\end{array}$} \\
\hline & & $\begin{array}{c}\text { natural } \\
\text { plantings }\end{array}$ & $\begin{array}{l}\text { trees planted } \\
\text { by man }\end{array}$ & $\begin{array}{c}\text { parks and other } \\
\text { types of } \\
\text { plantings }\end{array}$ & $\begin{array}{l}\text { occurrence } \\
\text { stability }\end{array}$ \\
\hline \multicolumn{2}{|c|}{ Number of research objects } & 7 & 10 & 6 & $100 \%$ \\
\hline 1. & Urtica dioica & 7 & 10 & 6 & 100,0 \\
\hline 2. & Geum urbanum & 7 & 8 & 4 & 82,6 \\
\hline 3. & Dactylis glomerata & 3 & 9 & 6 & 78,2 \\
\hline 4. & Galium aparine & 7 & 8 & 3 & 78,2 \\
\hline 5. & Poa pratensis & 2 & 9 & 5 & 69,5 \\
\hline 6. & Mycelis muralis & 3 & 7 & 5 & 65,2 \\
\hline 7. & Poa nemoralis & 3 & 8 & 4 & 65,2 \\
\hline 8. & Impatiens parviflora & 7 & 6 & 2 & 65,2 \\
\hline 9. & Taraxacum officinale & 1 & 10 & 3 & 60,8 \\
\hline 10. & Fragaria vesca & 1 & 9 & 3 & 56,5 \\
\hline 11. & Hypericum perforatum & 2 & 7 & 3 & 52,1 \\
\hline 12. & Phleum pratense & 1 & 5 & 5 & 47,8 \\
\hline 13. & Glechoma hederacea & 4 & 5 & 2 & 47,8 \\
\hline 14. & Rumex acetosa & 2 & 4 & 4 & 43,4 \\
\hline 15. & Anemone nemorosa & 4 & 2 & 4 & 43,4 \\
\hline
\end{tabular}




\begin{tabular}{|c|c|c|c|c|c|}
\hline 16. & Alopecurus pratensis & 1 & 6 & 3 & $\mathbf{4 3 , 4}$ \\
\hline 17. & Rubus idaeus & 5 & 4 & 1 & 43,4 \\
\hline 18. & Arctium lappa & 3 & 3 & 3 & 39,1 \\
\hline 19. & Stellaria media & 2 & 5 & 2 & 39,1 \\
\hline 20. & Cirsium arvense & 3 & 4 & 2 & 39,1 \\
\hline 21. & Agropyron repens & 1 & 5 & 2 & 34,7 \\
\hline 22. & Leucanthemum vulgare & 1 & 5 & 2 & 34,7 \\
\hline 23. & Artemisia vulgaris & 2 & 4 & 2 & 34,7 \\
\hline 24. & Dryopteris filix-mas & 2 & 4 & 2 & 34,7 \\
\hline 25. & Ficaria verna & 4 & 2 & 2 & 34,7 \\
\hline 26. & Deschampsia caespitosa & 2 & 3 & 2 & 30,4 \\
\hline 27. & Potentilla anserina & 2 & 3 & 2 & 30,4 \\
\hline 28. & Lysimachia nummularia & 3 & 1 & 2 & 26,0 \\
\hline 29. & Oxalis acetosella & 3 & 1 & 2 & 26,0 \\
\hline 30. & Medicago lupulina & 1 & 4 & 1 & 26,0 \\
\hline 31. & Lupinus polyphyllus & 2 & 3 & 1 & 26,0 \\
\hline 32. & Stellaria graminea & 2 & 3 & 1 & 26,0 \\
\hline 33. & Tanacetum vulgare & 2 & 3 & 1 & 26,0 \\
\hline 34. & Veronica chamaedrys & 2 & 3 & 1 & 26,0 \\
\hline 35. & Equisetum sylvaticum & 3 & 2 & 1 & 26,0 \\
\hline 36. & Heracleum sibiricum & 3 & 2 & 1 & 26,0 \\
\hline 37. & Bromus inermis & 1 & 3 & 1 & 21,7 \\
\hline 38. & Chelidonium majus & 1 & 3 & 1 & 21,7 \\
\hline 39. & Equisetum arvense & 1 & 3 & 1 & 21,7 \\
\hline 40. & Vicia villosa & 1 & 3 & 1 & 21,7 \\
\hline 41. & Carex hirta & 2 & 2 & 1 & 21,7 \\
\hline 42. & Ranunculus acris & 2 & 2 & 1 & 21,7 \\
\hline 43. & Gagea lutea & 1 & 1 & 2 & 17,4 \\
\hline 44. & Tussilago farfara & 1 & 1 & 2 & 17,4 \\
\hline 45. & Agrimonia eupatoria & 1 & 2 & 1 & 17,4 \\
\hline 46. & Arrhenatherum elatius & 1 & 2 & 1 & 17,4 \\
\hline 47. & Galium verum & 1 & 2 & 1 & 17,4 \\
\hline 48. & Medicago sativa & 1 & 2 & 1 & 17,4 \\
\hline 49. & Plantago lanceolata & 1 & 2 & 1 & 17,4 \\
\hline 50. & Rumex obtusifolius & 2 & 1 & 1 & 17,4 \\
\hline 51. & Stellaria holostea & 2 & 1 & 1 & 17,4 \\
\hline 52. & Caltha palustris & 1 & 1 & 1 & 13,0 \\
\hline 53. & Carex pallescens & 1 & 1 & 1 & 13,0 \\
\hline 54. & Geum rivale & 1 & 1 & 1 & 13,0 \\
\hline 55. & Galium mollugo & 1 & 4 & - & 21,7 \\
\hline 56. & Fragaria moschata & 3 & 2 & - & 21,7 \\
\hline 57. & Chamaenerion angustifolium & 1 & 3 & - & 17,4 \\
\hline 58. & Equisetum pratense & 1 & 3 & - & 17,4 \\
\hline 59. & Bromus hordeaceus & 2 & 2 & - & 17,4 \\
\hline 60. & Lathyrus pratensis & 2 & 2 & - & 17,4 \\
\hline 61. & Iris pseudacorus & 3 & 1 & - & 17,4 \\
\hline 62. & Solanum dulcamara & 3 & 1 & - & 17,4 \\
\hline 63. & Epilobium montanum & 1 & 2 & - & 13,0 \\
\hline 64. & Galeopsis tetrahit & 1 & 2 & - & 13,0 \\
\hline 65. & Knautia arvensis & 1 & 2 & - & 13,0 \\
\hline 66. & Scirpus sylvaticus & 1 & 2 & - & 13,0 \\
\hline 67. & Achillea collina & 2 & 1 & - & 13,0 \\
\hline 68. & Aegopodium podagraria & 2 & 1 & - & 13,0 \\
\hline 69. & Alchemilla monticola & 2 & 1 & - & 13,0 \\
\hline 70. & Alyssum alyssoides & 1 & 1 & - & 8,5 \\
\hline 71. & Astragalus glycyphyllus & 1 & 1 & - & 8,5 \\
\hline
\end{tabular}




\begin{tabular}{|c|c|c|c|c|c|}
\hline 72. & Bidens cernua & 1 & 1 & - & 8,5 \\
\hline 73. & Carex vulpina & 1 & 1 & - & 8,5 \\
\hline 74. & Circaea lutetiana & 1 & 1 & - & 8,5 \\
\hline 75. & Cirsium oleraceum & 1 & 1 & - & 8,5 \\
\hline 76. & Dianthus deltoides & 1 & 1 & - & 8,5 \\
\hline 77. & Lathyrus tuberosus & 1 & 1 & - & 8,5 \\
\hline 78. & Linaria vulgaris & 1 & 1 & - & 8,5 \\
\hline 79. & Phalaris arundinacea & 1 & 1 & - & 8,5 \\
\hline 80. & Prunella vulgaris & 1 & 1 & - & 8,5 \\
\hline 81. & Senecio jacobaea & 1 & 1 & - & 8,5 \\
\hline 82. & Silene vulgaris & 1 & 1 & - & 8,5 \\
\hline 83. & Trifolium medium & 1 & 1 & - & 8,5 \\
\hline 84. & Vicia angustifolia & 1 & 1 & - & 8,5 \\
\hline 85. & Angelica sylvestris & 4 & - & 1 & 21,7 \\
\hline 86. & Ranunculus repens & 3 & - & 1 & 17,4 \\
\hline 87. & Solidago virgaurea & 1 & - & 2 & 13,0 \\
\hline 88. & Galeobdolon luteum & 2 & - & 1 & 13,0 \\
\hline 89. & Maianthemum bifolium & 2 & - & 1 & 13,0 \\
\hline 90. & Rubus caesius & 2 & - & 1 & 13,0 \\
\hline 91. & Actaea spicata & 1 & - & 1 & 8,5 \\
\hline 92. & Anemone ranunculoides & 1 & - & 1 & 8,5 \\
\hline 93. & Euphorbia cyparissias & 1 & - & 1 & 8,5 \\
\hline 94. & Lythrum salicaria & 1 & - & 1 & 8,5 \\
\hline 95. & Potentilla argentea & 1 & - & 1 & 8,5 \\
\hline 96. & Trifolium repens & - & 5 & 4 & 39,1 \\
\hline 97. & Anchusa officinalis & - & 5 & 1 & 26,0 \\
\hline 98. & Bellis perennis & - & 4 & 1 & 21,7 \\
\hline 99. & Campanula glomerata & - & 1 & 2 & 13,0 \\
\hline 100. & Trifolium campestre & - & 1 & 2 & 13,0 \\
\hline 101. & Hieracium pilosella & - & 2 & 1 & 13,0 \\
\hline 102. & Myosotis sylvatica & - & 2 & 1 & 13,0 \\
\hline 103. & Plantago major & - & 2 & 1 & 13,0 \\
\hline 104. & Campanula rotundifolia & - & 1 & 1 & 8,5 \\
\hline 105. & Chenopodium album & - & 1 & 1 & 8,5 \\
\hline 106. & Cichorium intybus & - & 1 & 1 & 8,5 \\
\hline 107. & Lathyrus pratensis & - & 1 & 1 & 8,5 \\
\hline 108. & Plantago media & - & 1 & 1 & 8,5 \\
\hline 109. & Veronica officinalis & - & 1 & 1 & 8,5 \\
\hline 110. & Galium palustre & 4 & - & - & 17,4 \\
\hline 111. & Myosoton aquaticum & 4 & - & - & 17,4 \\
\hline 112. & Alopecurus aequalis & 2 & - & - & 8,5 \\
\hline 113. & Athyrium filix-femina & 2 & - & $=$ & 8,5 \\
\hline 114. & Dryopteris carthusiana & 2 & - & - & 8,5 \\
\hline 115. & Erigeron acris & 2 & - & - & 8,5 \\
\hline 116. & Galium odoratum & 2 & - & - & 8,5 \\
\hline 117. & Glyceria fluitans & 2 & - & - & 8.5 \\
\hline 118. & Impatiens noli-tangere & 2 & - & - & 8,5 \\
\hline 119. & Lamium purpureum & 2 & - & - & 8,5 \\
\hline 120. & Lysimachia vulgaris & 2 & - & - & 8,5 \\
\hline 121. & Phragmites communis & 2 & - & $=$ & 8,5 \\
\hline 122. & Platanthera bifolia & 2 & - & $=$ & 8,5 \\
\hline 123. & Polypodium vulgare & 2 & - & - & 8,5 \\
\hline 124. & Trifolium arvense & 2 & - & - & 8.5 \\
\hline 125. & Anthriscus sylvestris & 1 & - & - & 4,3 \\
\hline 126. & Carex pseudocyperus & 1 & - & $=$ & 4,3 \\
\hline 127. & Carex spicata & 1 & - & 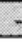 & 4,3 \\
\hline
\end{tabular}




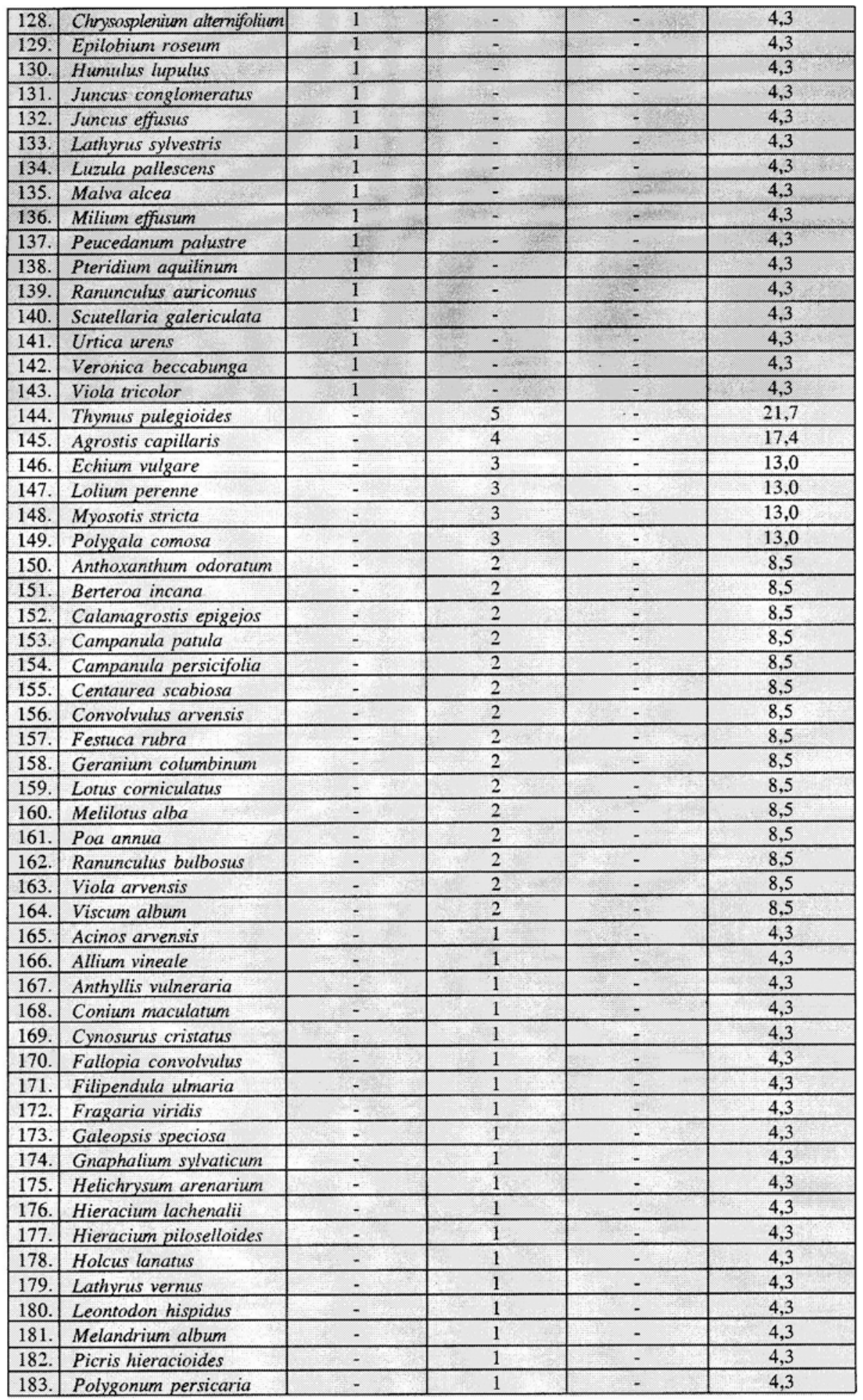




\begin{tabular}{|r|l|c|c|c|c|}
\hline 184. & Ranunculus cassubicus & - & 1 & - & 4,3 \\
\hline 185. & Rumex crispus & - & 1 & - & 4,3 \\
\hline 186 & Sedum acre & - & 1 & - & 4,3 \\
\hline 187. & Sedum maximum & - & 1 & - & 4,3 \\
\hline 188. & Sonchus oleraceus & - & 1 & - & 4,3 \\
\hline 189. & Trifolium montanum & - & 1 & - & 4,3 \\
\hline 190. & Trifolium pratense & - & 1 & - & 4,3 \\
\hline 191. & Vaccinium myrtillus & - & 1 & - & 4,3 \\
\hline 192. & Vicia hirsuta & - & 1 & - & 4,3 \\
\hline 193. & Vicia sepium & - & 1 & - & 4,3 \\
\hline 194. & Vicia tetrasperma & - & 1 & - & 4,3 \\
\hline 195. & Viscaria vulgaris & - & 1 & - & 4,3 \\
\hline 196. & Hepatica nobilis & - & - & 2 & 8,5 \\
\hline 197. & Hedera helix & - & - & 1 & 4,3 \\
\hline 198. & Hemerocallis fulva & - & - & 1 & 4,3 \\
\hline 199. & Lilium bulbiferum & - & - & 1 & 4,3 \\
\hline 200. & Narcissus poëticus & - & - & 1 & 4,3 \\
\hline 201. & Vinca minor & - & - & 1 & 4,3 \\
\hline
\end{tabular}

Fig. 1. Location of the objects studied (original)

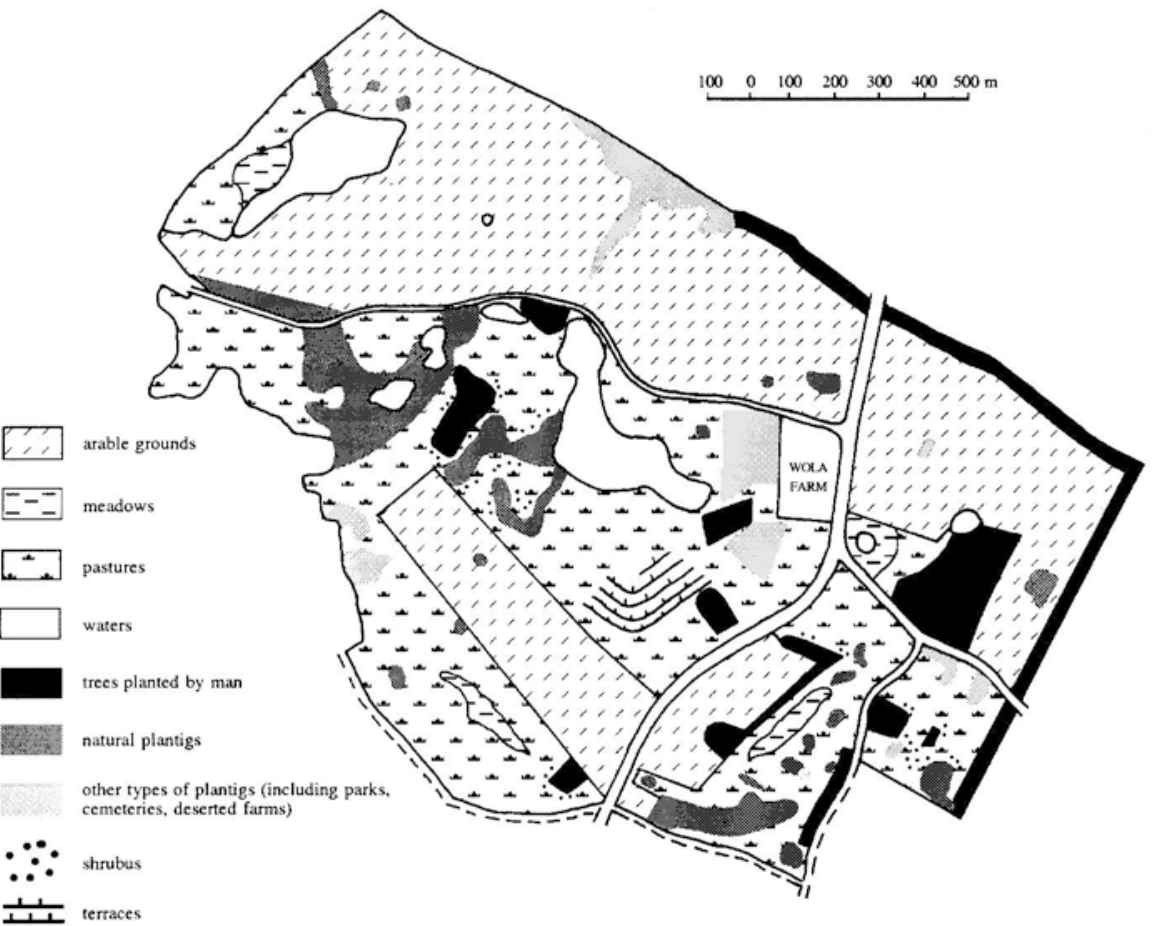


Fig. 2. Geographical analysis of flora in the objects studied

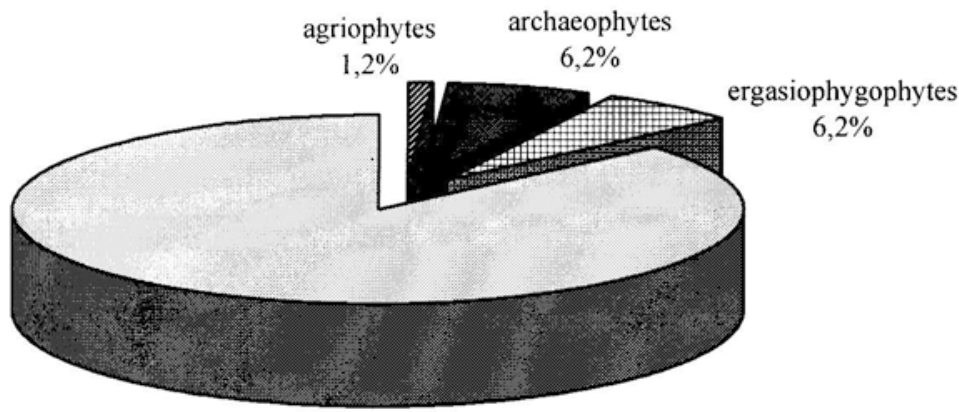

apophytes

$86,4 \%$

Fig. 3. Origin of apophytes in flora of plantings

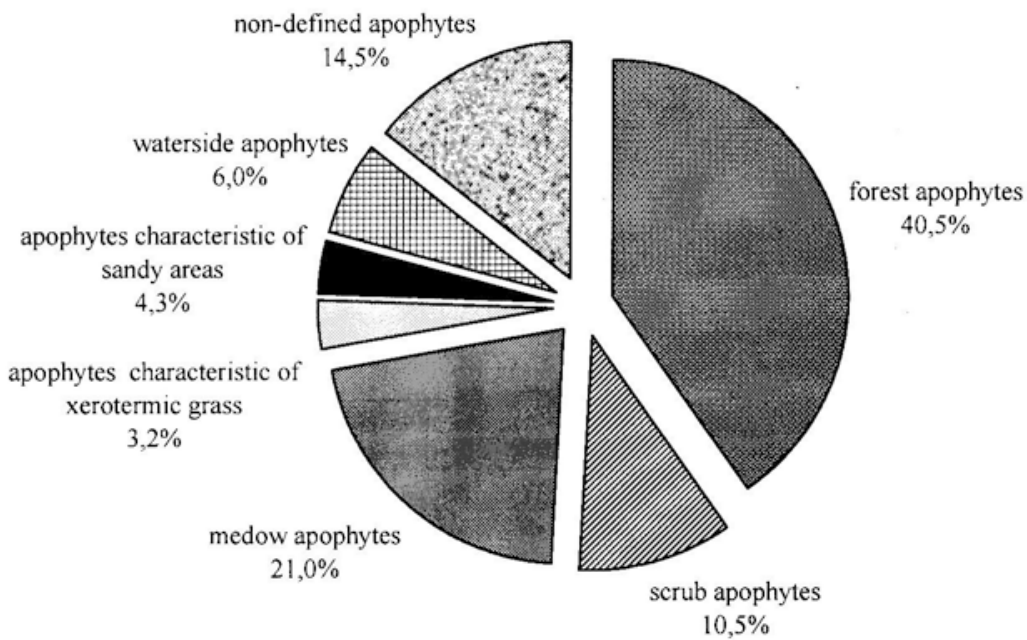

Fig. 4. Life forms of plants

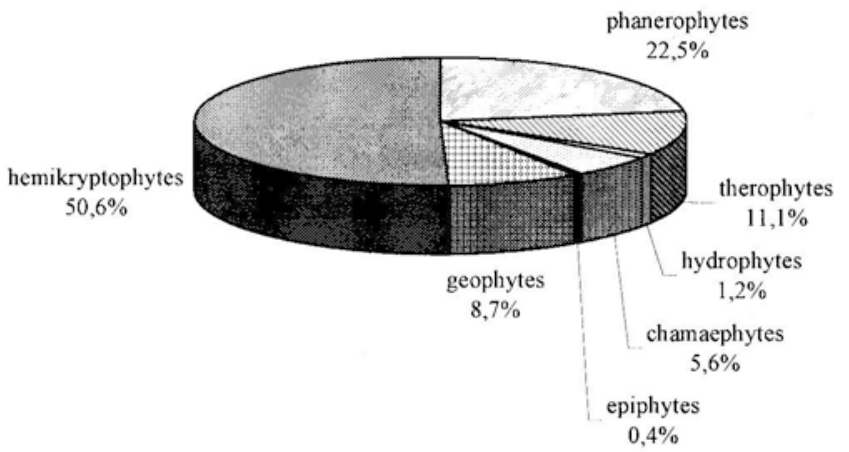


Fig. 5. Number of taxons presents in plantings compared with the segetal flora of NorthEstern Poland

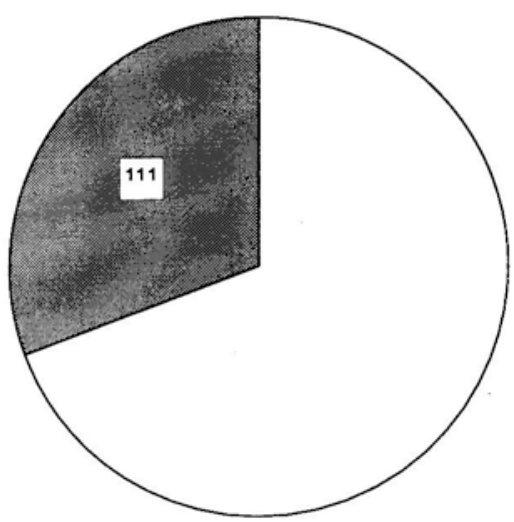

Segetal flora of North-Eastern Poland $=361$ species (Korniak 1992)

segetal flora of the objects studied

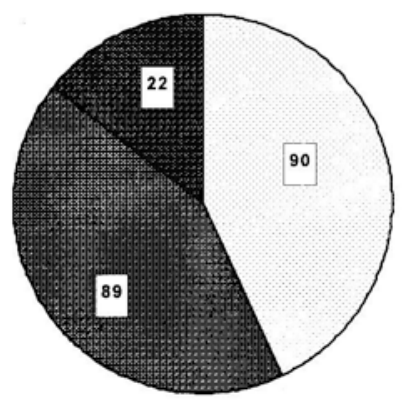

Flora of field plantings $=201$ species

閶 very common and common segetal species

very rare and rare segetal species

non-synanthropic spontaneophytes

Fig. 6. Global index of occurrence frequency of very common and common weeds in field plantings

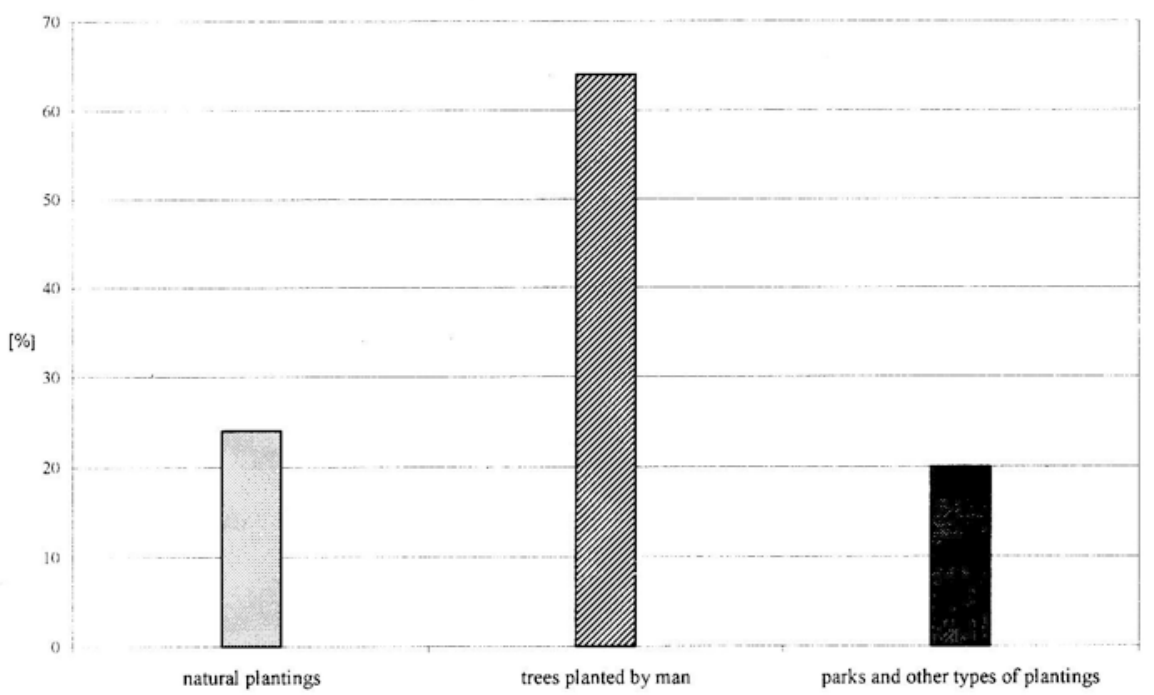

